\title{
Use of Burpees in Combat Sports Conditioning Training - A Pilot Study
}

\author{
Luboslav Siska ${ }^{1}$, Jaroslav Brodani ${ }^{1}$ \\ ${ }^{1}$ Department of physical education and sport, Faculty of education, Constantine the philosopher Universit in \\ Nitra, Trieda Andreja Hlinku 1, 94974 Nitra, Slovak Republic
}

*Corresponding Author: Luboslav Siska, Department of physical education and sport, Faculty of education, Constantine the philosopher Universit in Nitra, Trieda Andreja Hlinku 1, 94974 Nitra, Slovak Republic

\begin{abstract}
The aim of our work was to design a specific endurance test, identical in duration to the boxing competition ( $3 \times 3 \mathrm{~min}$ ) and develop an assessment methodology. The essence of the test was to do one repetition of the Burpee at regular intervals triggered by a visual signal, which was signaled every 3 seconds using the FiTRO agility system. In this way, the athlete carried out 35 burpees in one round ( 3 min.), i.e. 105 burpees throughout the duration of the test. In terms of assessment, we measured the individual burpees in milliseconds ( $m s$ ) along with the drop of performance, which was reflected by an increase in time as a percentage value. The intensity of load was monitored through heart rate and blood lactate. The average duration of a burpee was $1960.4 \mathrm{~ms}$ in the first round, $2050.2 \mathrm{~ms}$ in the second round and $2207.4 \mathrm{~ms}$ in the third round, and we also identified statistically significant differences in the mean values between the retries in each round ( $p<.01$ ). The decrease in performance (index of fatigue) was $7.54 \%$ in the first round, $15.29 \%$ in the second round at $17.52 \%$ in the third round, and the total was $22.28 \%$. The maximum heart rate reached $164 \mathrm{bpm}$, representing 93.18\% of the maximum value. The level of lactate in the 4th minute after the load was $14.1 \mathrm{mmol}$ and it dropped to $8.6 \mathrm{mmol} 15$ minutes after the load. We managed to create a short exercise program that can be used as a training and diagnostic tool. The decrease in performance, which is noticeable in a real match, was also demonstrated during the test. Therefore, it is necessary to carry out a deeper analysis of the relationship of the possible better results in the test as a determining factor of success in the match.
\end{abstract}

Keywords: Boxing, Endurance, Burpee, Decrease in Performance, Lactate, Heart Rate

\section{INTRODUCTION}

High sports performance, which can nowadays be observed in combat sports, is the result of a continuous training process and use of specialized means and methods (Šiška, 2016). In terms of endurance, the combatant must be able to repeatedly perform explosive moves throughout the duration of the match with the least possible drop in performance, while the internal response of the body in the case of blood lactate level stays in the range of 11-16 mmol and the heart rate stays in the sub-maximum to maximum range (Ghosh, 2010; Arsenau, 2011; Oeurgui, 2015). The explosive moves are of a different nature, but according to the available studies, the alternation of high-intensity and lower-intensity intervals is approximately in the ratio of 1:1 to $1: 2$ and the duration of the highintensity interval is 1-2s on average (Šiška, 2016; Oeurgui, 2014). Many authors dealt with the idea of creating a short movement program in the time duration of a competitive match, which would be suitable for use as a training resource for the development of endurance needed for combat sports in the standing position and using punches and kicks. Several variations of monitoring of internal and external response of the organism to specific exercises and then comparing it with the competitors or the training game were developed (Oeurgui, 2015; Hatfield, 2003; Nunan, 2006). The problem in these programs is the impossibility of immediate monitoring of intensity and decrease in performance during the load. Since in conditioning training it is recommended to use multiple-joint exercises, this condition is met by the Burpee in terms of time, which was used as a diagnostic tool in a number of studies, however, only as to the number of repetitions (Podstawski, 2016; Podstawski, 2016; Moura, 2016). After a slight modification, the select exercise can also be quantified (determine the exact time 
of the duration of the entire exercise) by using the FiTRO agility system (fitronic.sk), which ensures the control of exercise intensity throughout the duration of the load. The aim of the work is to design an endurance load, which can be used as a training and diagnostic tool, which allows us to precisely determine the drop in performance.

\section{MATERIALS AND METHODS}

The research was attended by a single sportsman aged 37, height $172 \mathrm{~cm}$, weight $70 \mathrm{~kg}$, HR max. $176 \mathrm{bpm}$. The total sports age of the sportsman is 30 years and he is a multiple Slovak champion in athletics and kickboxing.

\subsection{Description of Test}

In terms of time, the test is identical to the timing of a boxing match, i.e. $3 \times 3$ minutes, with a 1 minute break between the rounds. A FITRO agility plate is placed on the wall at a height of $1.2 \mathrm{~m}$ from the ground. In the immediate vicinity of the platform there is a PC showing the signals to perform the exercise. At a distance of $1 \mathrm{~m}$ from the wall there is a line, which serves as a starting position (fig. 1). The essence of the test was to do one repetition of the Burpee at regular intervals triggered by a visual signal, which was signaled every 3 seconds using the FiTRO agility system. The exercise is modified - the final jump stage is replaced by a step-out accompanied by hitting the plate by hand. In one round, the sportsman performs 35 burpees, which gives us about 3 minutes at the 3 second interval and addition of the time of the individual repetitions. This is followed by a 1 minute break, and 35 signals representing the second round. Overall, the sportsman does 3 rounds with 105 burpees. The test evaluates the execution speed of the exercise in milliseconds with focus on the increasing time, which represents the index of fatigue. The tracking of internal response of the sportsman's body was carried out by the SUNTO heart rate monitoring device, and the blood lactate level was measured by LACTATE SCOUT in the 4th and 15th minute after the load.

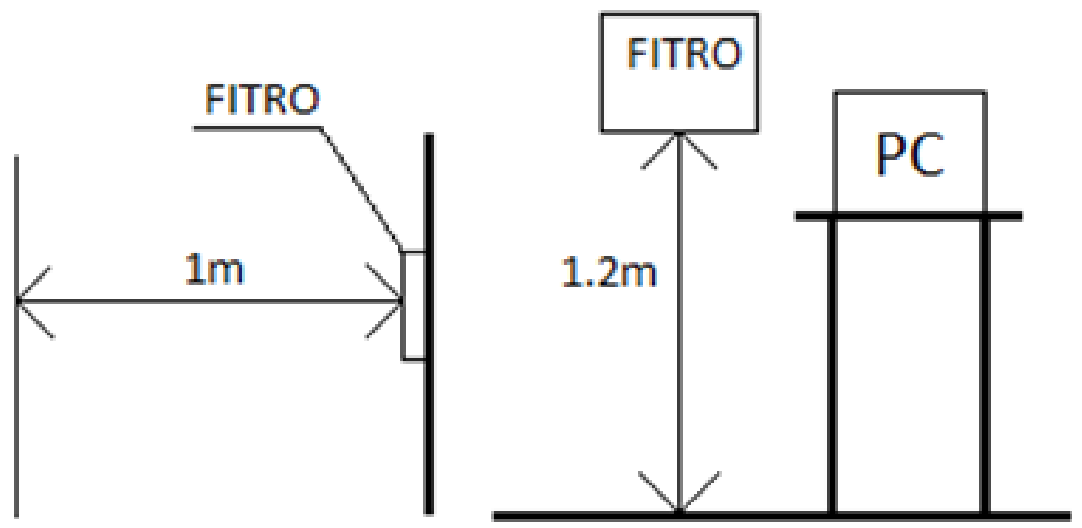

Fig1. Visual Representation of the Test

The results reported in the test are described using the basic descriptive statistics "average time value of exercise" in each of the rounds, standard deviation, minimum value, maximum value and visual representation using box charts. The statistically significant differences in the mean values between the rounds were expressed by a T-test at the 5\% and $1 \%$ level of significance. The substantive significance was assessed by the Cohen's "d" effect size coefficient. The subsequent time data for each repetition were displayed in a line chart and overlaid by a linear trend line. The performance drop (index of fatigue) is expressed as a percentage difference of the maximum and minimum values on the linear trend line with regard to the minimum value.

\subsection{Results}

Table1. Descriptive Statistics

\begin{tabular}{|c|c|c|c|c|c|}
\hline \multicolumn{2}{|c|}{ I. ROUND } & \multicolumn{2}{c|}{ II. ROUND } & \multicolumn{2}{c|}{ III. ROUND } \\
\hline M & 1960,43 & M & 2050,26 & M & 2207,43 \\
\hline SD & 68,17 & SD & 102,91 & SD & 119,47 \\
\hline Min & 1853 & Min & 1876 & Min & 1979 \\
\hline Max & 2110 & Max & 2300 & Max & 2493 \\
\hline
\end{tabular}


Performance varied during the test. The most consistent values were recorded in the first round, which was manifested by the smallest standard deviation. The average performance worsened in each round: between I. and II. Round, it worsened by about $90 \mathrm{~ms}$ and between II. and III. round, it worsened by $157 \mathrm{~ms}$ (Tab. 1). The best performance was recorded in the first round. The decrease in performance was most evident in the third round, and it was reflected by the worst average performance and highest standard deviation (Fig. 2).

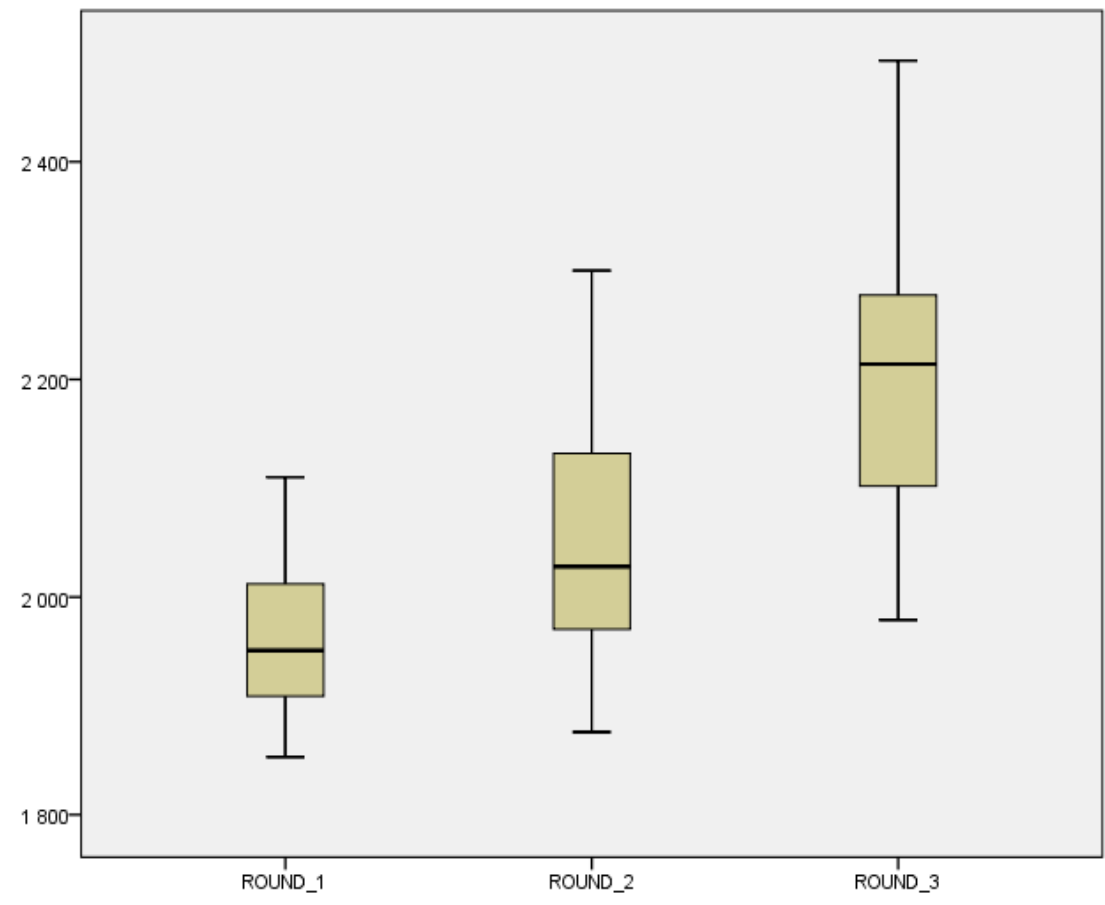

Fig2. Box graphs with the measured values

We identified statistically significant differences in the mean values and effect size coefficients "d" between:

I. and II round $\mathrm{t}(34)=-7,91, \mathrm{p}<.01,, \mathrm{~d}^{\mathrm{c}}=1,03$

I. and III. round $\mathrm{t}(34)=-14,98, \mathrm{p}<.01,, \mathrm{~d}^{\prime \prime}=2,54$

II. and III. round $\mathrm{t}(34)=-12,61, \mathrm{p}<.01,, \mathrm{~d}^{“}=1,41$

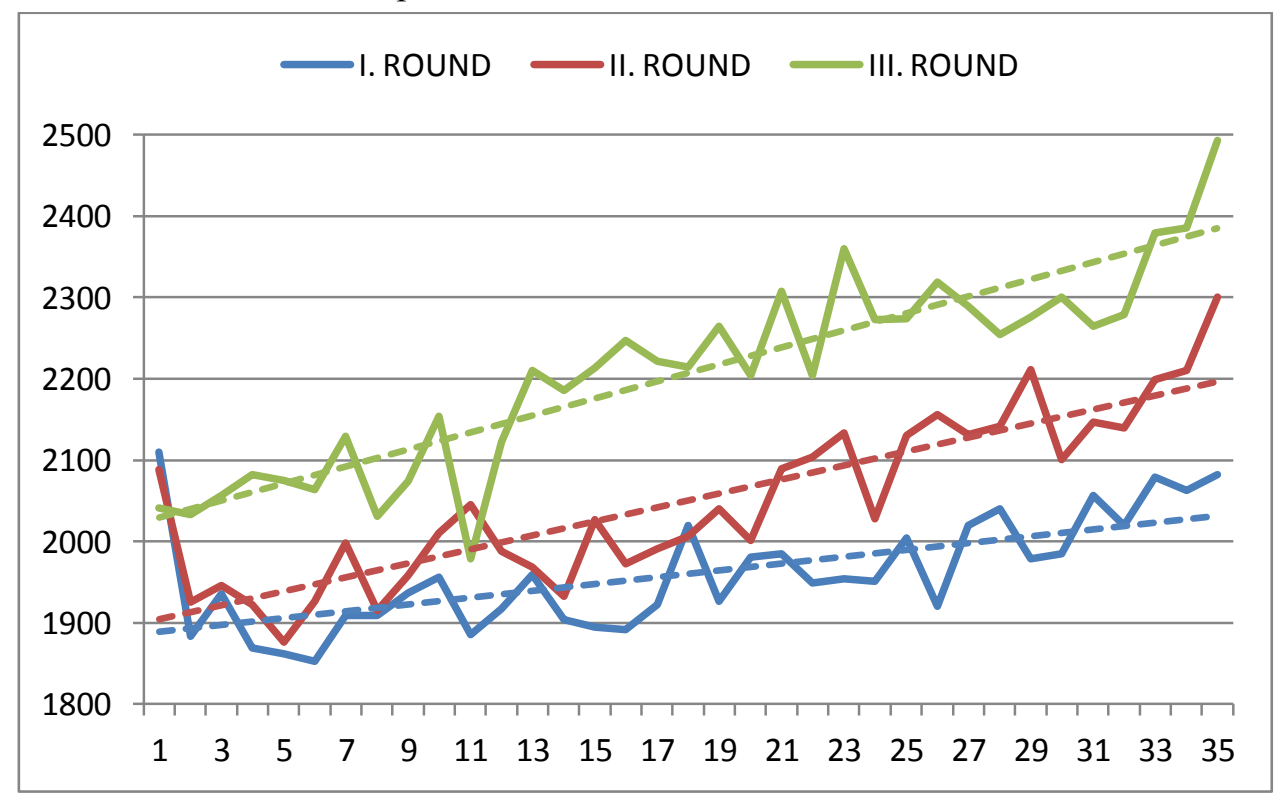

Fig3. Time values of the individual repetitions and their linear trending

Percentage of decrease in performance (fatigue index) in each round:

\section{I.KOLO - 7.54\% II. KOLO - 15.29\% III. KOLO - 17.52\%}


The total decrease in performance for all three rounds was at the level $\mathbf{2 2 . 2 8 \%}$ (fig.4).

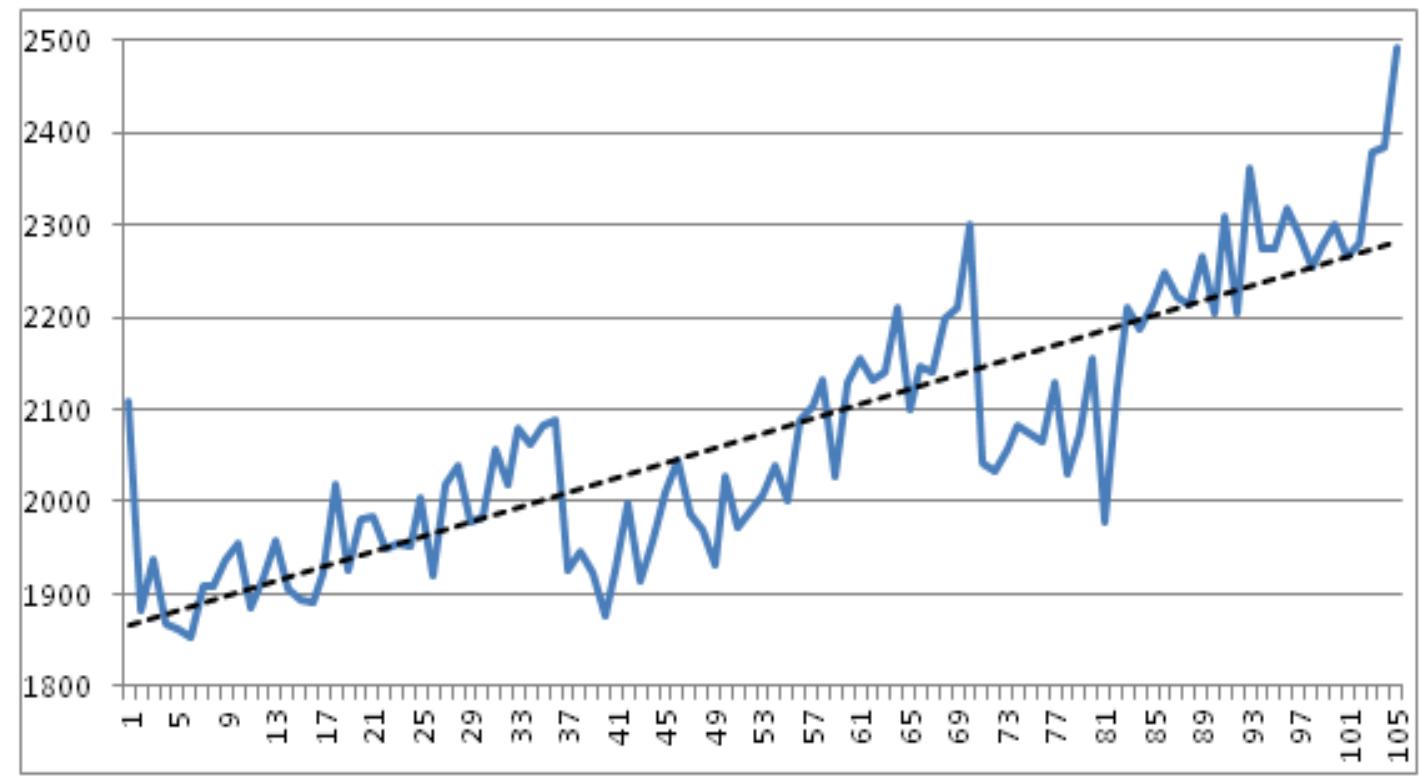

Fig4. Display of all time values and total fatigue index

The heart rate gradually increased in each round. In the first round, it reached $160 \mathrm{bpm}$ and it subsequently decreased during the break by $24 \mathrm{bpm}$ to 136 . In the second round, there was an increase to $163 \mathrm{bpm}$ and an even greater drop by $1 \mathrm{bpm}$ during the break at $135 \mathrm{bpm}$ compared to the previous round. The highest value was recorded in the third round at $164 \mathrm{bpm}$, whereby this value represented 93.18\% of the maximum measured value in the sportsman (Fig. 4).

The blood lactate reached the value of $14,1 \mathrm{mmol}$ in the 4 th minute after the load and it dropped to $8.6 \mathrm{mmol}$ in the 15 th minute.

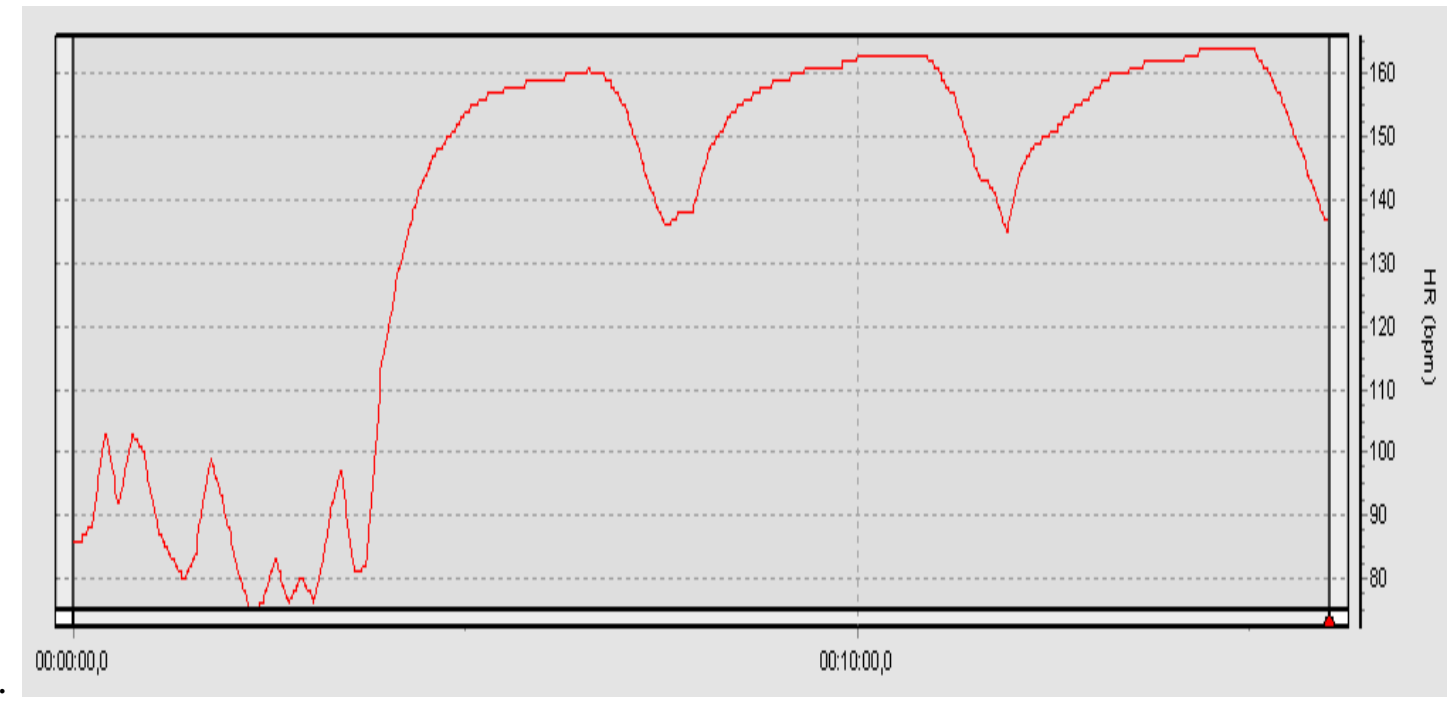

Fig5. Course of Heart Rate During Exercise

\section{DISCUSSION}

In our research, we tried to propose a tool both for sports training and diagnostics. In terms of conditioning training, a prerequisite for success in a competitive match is the sportsman's ability to minimize fatigue, which can be accomplished by an appropriately designed training program. We focused on the assessment of the index of fatigue through increasing the time required to perform one burpee, which is a unique research endeavor in this area, therefore, we have no results to compare it with. As regards the internal response of the body to load, we reached similar values of blood lactate and heart rate as those reported by other authors for specific boxing exercises, sparring, exercises or competitive matches (Ghosh, 2010; Arsenau, 2011; Oeurgui, 2015). In terms of subjective evaluation, 
the effort required to perform the burpee was comparable to performing an attack in a competitive match mainly in the third round. The accumulated fatigue was felt to a large extent, particularly when transitioning from the push-up to stand. Since the exercise is carried out on a visual signal, a certain level of response capabilities is assumed, which can also have an effect on the resulting time. In the future, however, it is necessary to analyze the fatigue in a greater detail, make a breakdown of the motion into its individual phases and determine the percentage of the individual phases in the overall time. Subsequently, it is necessary to determine in which of the stages fatigue manifests itself the fastest, including the initial responses to the visual signal. In our case, the visual signals to trigger the burpee were generated at regular intervals of three seconds, which corresponds to the ratio between the active and inactive phases from previous research (Šiška, 2016), however, since we cannot predict the number, length and period of these phases in a real boxing match, and the FiTRO device also offers us the possibility of random signals in any interval, one also needs to focus on this possibility and the use of other devices.

\section{COnClusions}

In conclusion, we can state that we managed to design a short power-endurance program, taking into account the intermittent nature of combat sports in a standing position and use of punches and kicks. We showed that the index of fatigue, which resulted in an increase in the time required to carry out the burpee and the internal response of the body during the load, has a similar development to the development in a real competitive match. The decrease in performance, which is noticeable in a real match, was also demonstrated during the test. Therefore, it is necessary to carry out a deeper analysis of the relationship of the possible better results in the test as a determining factor of success in the match. We recommend using the proposed program in the sports preparation with the aim to improve the power-endurance skills for combat sports.

\section{ACKNOWLEDGMENTS}

The project was supported by the VEGA 1/0931/16 grant titled: The psycho-physiological diagnostic of stress considering pre - competing physical state in combat sports.

\section{REFERENCES}

[1] Šiška L', Brod’áni J. (2016). Analysis of a Boxing match - A pilot study. J Phys Ed Sport, 16(4), Art 178, pp. $1111-1114$.

[2] Šiška L, Brod’áni J, Štefanovký M, Todorov S. (2016). Basic reliability parameters of a boxing punch. J Phys Ed Sport, 16(1), Art 38, pp. 241 - 244.

[3] Davis P, Wittekind A, Beneke R. (2013). Amateur Boxing: Activity Profile of Winners and Losers. International Journal of Sports Physiology and Performance, 8, 84-91.

[4] Arseneau E, Mekary S, Leger L. (2011). VO2 requirements of boxing exercises. J Strength Cond Res, $25(2)$ 348-359.

[5] Ghosh A. (2010).Heart Rate, Oxygen Consumption and Blood Lactate Responses During Specific Training in Amateur Boxing. International Journal of Applied Sports Sciences, 22 (1), 1-12.

[6] Hatfield F. (2003).General Points Of Conditioning For Boxers. on-line: http://www.bodybuilding. com/fun/luis14.htm,

[7] Ouergui I, Houcine N, Marzouki H, Davis P, Zaouali M, Franchini E, et al. (2015).Development of a Noncontact Kickboxing Circuit Training Protocol That Simulates Elite Male Kickboxing Competition. J Strength Cond Res, 29 (12), 3405-3411. 12.

[8] Nunan D. (2006). Development of a Sports Specific Aerobic Capacity Test for Karate - A Pilot Study. J Sports Sci Med, 5(CSSI): 47-53.

[9] Podstawski R, Markowski P, Choszcz D et al. (2016).Methodological aspect of evaluation of the reliability the 3-Minute Burpee Test. Arch Budo Sci Martial Art Extreme Sport, 12:137-144

[10] Podstawski R, Markowski P, Choszcz D et al. (2016).Correlations between anthropometric indicators, heart rate and endurance-strength abilities during high-intensity exercise of young women. Arch Budo Sci Martial Art Extreme Sport, 12: 17-24

[11] Ouergui, I., Hassin, N., Haddad, N., Franchini, E., Behm, G. D., Wong, P. D., Gmada, N., Bouhlel, E.. (2014). Time - motion analysis of elite male kickboxing competition.Journal of Strength and Conditioning Research, 28(12) 3537-3543. 
[12] Ratamess, NA, Rosenberg, JG, Klei, S, Dougherty, BM, Kang, J, Smith, CR, Ross, RE, and Faigenbaum, AD. (2015). Comparison of the acute metabolic responses to traditional resistance, body-weight, and battling rope exercises. J Strength Cond Res, 29(1): 47-57.

[13] Moura FC, Machado AAN, Vieira LL, Abreu ES, Soares PM, Brito GAC, CarlosPS, Costa EE. ( 2016).Jiu-jitsu athletes' cardiovascular responses in an adapted burpee test. Asian Journal of Science and Technology, 7(1): 2208-221.

[14] http://www.fitronic.sk/ (FiTRO agility check)

Citation: Luboslav, Siska, and Brodani Jaroslav. "Use of Burpees in Combat Sports Conditioning Training A Pilot Study." International Journal of Sports and Physical Education (IJSPE), vol 3, no. 4, 2017, pp. 1-6. doi:http://dx.doi.org/10.20431/2454-6380.0304001.

Copyright: (C) 2017 Authors. This is an open-access article distributed under the terms of the Creative Commons Attribution License, which permits unrestricted use, distribution, and reproduction in any medium, provided the original author and source are credited. 